\title{
On the top-antitop invariant mass spectrum at the LHC from a Higgs boson signal perspective
}

\author{
S. Moretti and D.A. Ross \\ School of Physics and Astronomy, University of Southampton, Highfield, Southampton SO17 1BJ, UK.
}

\begin{abstract}
We investigate the effect of one-loop corrections of $\mathcal{O}\left(\alpha_{S}^{2} \alpha_{W}\right)$ on the $t \bar{t}$ invariant mass spectrum at the Large Hadron Collider (LHC) in presence of both resonant and non-resonant Higgs boson effects. We show that corrections of $\mathcal{O}\left(\alpha_{S}^{2} \alpha_{W}\right)$ involving a non-resonant Higgs boson are comparable to or even larger than those involving interference with the $s$-channel resonant Higgs boson amplitude and that both of these are subleading with respect to all other (non-Higgs) diagrams through that order. We also compute the contribution through $\mathcal{O}\left(\alpha_{S}^{2} \alpha_{W}^{2}\right)$ of resonant Higgs boson production (i.e. Higgs production via $g g$ fusion) as well as the pure QCD ones of $\mathcal{O}\left(\alpha_{S}^{3}\right)$. Altogether, we show that the well known peak-dip structure of the $M_{t \bar{t}}$ spectrum emerging from interference effects between the $t, u$-channel $g g$-induced Leading Order (LO) QCD diagrams and the one due to a Higgs boson in $s$-channel via $g g$-fusion is drastically swamped by the remainder of the terms of $\mathcal{O}\left(\alpha_{S}^{2} \alpha_{W}\right)$ discussed above.
\end{abstract}

\section{Introduction}

Higgs decay to top-antitop pairs could be a search channel in a variety of models where the corresponding Branching Ratio (BR) becomes dominant, owing to the fact that, in some such models, the competing Higgs decays into $W^{+} W^{-}$and $Z Z$ pairs can be suppressed or non-existent. For example, in the case of the Minimal Supersymmetric Standard Model (MSSM), this naturally occurs if the decaying Higgs boson is the CP-odd one, $A$, but also for the heavy $\mathrm{CP}$-even one, $H$, when $\cos (\beta-\alpha) \approx 0$ (see 1] for the definition of $\alpha$ and $\beta$ and the relevant MSSM Feynman rules). In this case, which is generally realised for $M_{A} \approx M_{H} \gtrsim 200 \mathrm{GeV}$, $A, H \rightarrow t \bar{t}$ becomes a feasible search channel [2]. Unfortunately, LEP (and now also Tevatron and LHC) limits almost exclude entirely the region of the $\left(M_{A}, \tan \beta\right)$ plane of the MSSM where significances above 5 can be achieved [3, 4]. On the other hand, in a generic 2-Higgs Doublet Model (2HDM), one can easily switch off the Higgs couplings to gauge boson pairs, as the trigonometric factor which controls this coupling is essentially a free parameter (unlike the case of the MSSM), whilst still being consistent with LEP (and more recent Tevatron and LHC) data [1]. Furthermore, even in those models where the scope of the Higgs $\rightarrow t \bar{t}$ decay channel may be limited, once a Higgs boson is found and its parameters (mass primarily, but possibly also width and discrete quantum numbers) measured, these can subsequently be exploited in the selection of the $t \bar{t}$ final state to render the Higgs visible [5]. This can be done, for example, for the purpose of measuring the corresponding $\mathrm{BR}$, thereby accessing the Higgs- $t \bar{t}$ coupling, in the attempt to profile the new resonance.

Therefore, it remains of the utmost importance to continue assessing the scope of existing colliders in extracting the Higgs $\rightarrow t \bar{t}$ signal, not least because, from a theoretical point of view, the Yukawa coupling between a Higgs boson and the top quark entering its rates is a parameter which generally plays a key role in the dynamics of any Beyond the Standard Model (BSM) scenario based on a Higgs mechanism: e.g., in its Electro-Weak Symmetry Breaking (EWSB) transition, in the Renormalisation Group Equations (RGEs) describing its evolution from high to low energy scales, etc. Finally, one should recall that the top(anti-top)-quark decays into a bottom(anti-bottom)-quark and a $W^{ \pm}$ boson rather than hadronising, thereby transmitting its spin properties to the decay products very

June 11, 2018 


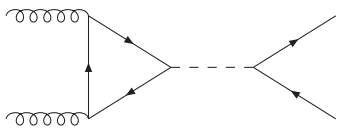

Figure 1: $g g \rightarrow t \bar{t}$ via a Higgs produced in the $s$-channel.

efficiently, so that the spin and CP nature of the Higgs boson(s) involved can be explored in suitable experimental observables [6, 7].

Clearly, in order to perform all the relevant searches for Higgs $\rightarrow t \bar{t}$ events, any source of SM corrections should be well-understood. This is true for the case of the Higgs signal, $g g \rightarrow$ Higgs $\rightarrow t \bar{t}$ [8, 9]. Regarding the QCD background, $q \bar{q}, g g \rightarrow t \bar{t}$, complete one-loop results exist for both the QCD [10] (see also [11]) and EW [12] (see also [13]) sectors. However, it is also of crucial importance to take into account interference effects between the above signal and background (limited to the $g g$ channel) [14].

Concerning such an interference, it is well known in the literature [14] that Higgs bosons produced via $g g$ fusion and decaying into top-antitop quark pairs interfere with the background $t, u$-channel LO QCD diagrams to produce a peak-dip structure in the $t \bar{t}$ invariant mass spectrum. This structure has been claimed as potentially observable at the LHC. We point out here that, if one properly includes through the perturbative order at which this effect emerges, i.e., $\mathcal{O}\left(\alpha_{S}^{2} \alpha_{W}\right)$, all other diagrams, such an effect is generally subleading with respect to those induced by all other (non-resonant) Higgs graphs appearing through that order and/or the ones due to all other (non-Higgs) diagrams, the latter being always the dominant contribution. (We neglect here the $q \bar{q} \rightarrow$ $t \bar{t}$ channel, as this is negligible at the LHC and does not involve a resonant Higgs contribution.)

The plan of the paper is as follows. In the next section we describe our calculation. In Sect. III we report on our numerical results. Finally, we conclude in Sect. IV.

\section{Calculation}

The computation exploited in this paper is described at length in Ref. [15], so we do not dwell here on the technical details. Rather, we just recall the salient features of the calculation, with a view at emphasising the role of the diagrams with an intermediate Higgs boson.
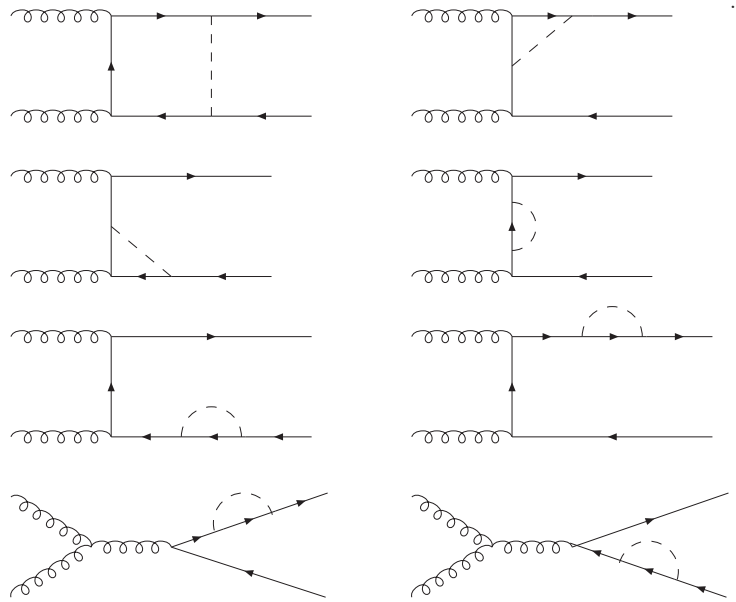

Figure 2: Remaining graphs involving a Higgs in the process $g g \rightarrow t \bar{t}$ (excluding gluon crossings).

For the complete EW corrections we need to consider all graphs containing a weakly (or electromagnetically) interacting internal particle. Graphs involving an internal Higgs are shown in Figs. 112 where Fig. 1 is the graph for the Higgs produced in the $s$-channel. This can interfere with the LO QCD amplitude $(t$ - and $u$-channel exchanges only since interference with $s$-channel exchange is forbidden by colour). Fig. 2 shows all the other graphs involving a Higgs, which can also interfere with (the complete) LO QCD amplitude. We want to compare the yield of these two interferences as well as to that of the entire set of EW corrections (the remaining graphs have the same topology as the graphs in Figs. 112 but with the Higgs replaced by a gauge and/or Goldstone boson.) The rates emerging through Next-to-LO (NLO) QCD will also be presented for reference. Notice that we only include here weak effects, thereby neglecting one-loop diagrams (and accompanying tree-level bremsstrahlung graphs) involving a photon. These were computed in [12] and found to be negligibly small. This is expected since QED corrections (after accounting for bremsstrahlung) see no large enhancement at the LHC, unlike the case of the weak ones, which are affected by large so-called Sudakov logarithms of $\mathcal{O}\left(\log ^{2}\left(\hat{s} / M_{W}^{2}\right)\right)$.

For the top mass and width we have taken $m_{t}=175 \mathrm{GeV}$ and $\Gamma_{t}=1.55 \mathrm{GeV}$, respectively. The $Z$ mass used was $M_{Z}=91.19 \mathrm{GeV}$ and was related to the $W$ mass, $M_{W}$, via the SM formula $M_{W}=M_{Z} \cos \theta_{W}$, where $\sin ^{2} \theta_{W}=0.232$. (Corresponding widths were $\Gamma_{Z}=2.5 \mathrm{GeV}$ and 
$\Gamma_{W}=2.08 \mathrm{GeV}$.) The Parton Distribution Functions (PDFs) that we have used are the CTEQ6 set [16] taken at the factorisation/renormalisation scale $Q=\mu=2 m_{t}$. (We also have checked other sets, but found no significant difference in the relative size of our corrections.) The choice of PDFs dictates the running and parameters used to compute $\alpha_{S}$, which has been taken at one-loop level in the calculation of terms of $\mathcal{O}\left(\alpha_{S}^{2}\right)$ and $\mathcal{O}\left(\alpha_{S}^{2} \alpha_{W}\right)$ (in conjunction with CTEQ6L1) and at two-loop level in the computation of effects of $\mathcal{O}\left(\alpha_{S}^{3}\right)$ (in conjunction with CTEQ6M). The value used throughout for the electromagnetic coupling was $\alpha=1 / 128$ (with $\alpha_{W}=\alpha / \sin \theta_{W}$ ). The NLO QCD corrections have been included through MCFM 17]. Finally, the Higgs decay widths have been computed by means of the programs described in Refs. 18] and [19]. All rates are presented at the LHC energy of $14 \mathrm{TeV}$, though the qualitative effects seen here would not change at 7 or at $8 \mathrm{TeV}$.

\section{Numerical results}

\subsection{CP-even Higgs state}

As the benchmark for the CP-even Higgs boson case we take the SM. Fig. 3 shows the contributions to the inclusive cross section from various different perturbative components. Clearly, the pure QCD contribution through $\mathcal{O}\left(\alpha_{S}^{3}\right)$ is largest (solid line). (In the plots it also incorporates the lowest order $\mathcal{O}\left(\alpha_{S}^{2}\right)$ term, dot-dashed line.) The $\mathcal{O}\left(\alpha_{S}^{2} \alpha_{W}\right)$ interference between the $t, u$-channel LO QCD diagrams with the one-loop $s$-channel Higgs graph is the dotted curve, which is always negative and largest for $M_{H}$ just above $2 m_{t}$. However, the dashed line, which refers to the interference with all graphs involving a Higgs boson, indicates that this term is actually positive and dominated by the graphs other than the $s$-channel Higgs exchange except in the region of $M_{H}$ between 250 and $550 \mathrm{GeV}$, where the interference is indeed negative but has an absolute value at least an order of magnitude smaller than that obtained from the $s$-channel Higgs exchange only. While this is a potential welcome feature through the $\mathcal{O}\left(\alpha_{S}^{2} \alpha_{W}\right)$, as it would increase the total signal yield, over a considerable range of $M_{H}$ (notice that the square of the $s$-channel Higgs graph through $\mathcal{O}\left(\alpha_{S}^{2} \alpha_{W}\right)$, diamond line, is consistently one order of magnitude or so smaller than its interference with the LO QCD terms), it becomes negligible if one includes all other non-Higgs

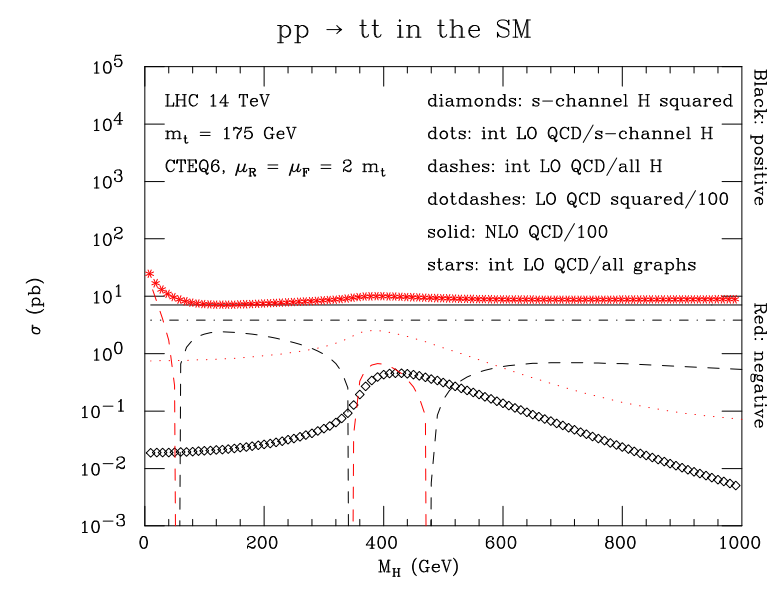

Figure 3: The integrated cross section at the LHC for all the components of the $g g \rightarrow t \bar{t}$ process described in the text. The Higgs processes are for the CP-even state of the SM. Where the curves are red it signifies that they correspond to a negative contribution (notice that the $y$-axis is logarithmic).

diagrams through $\mathcal{O}\left(\alpha_{S}^{2} \alpha_{W}\right)$. In fact, the complete result at such a perturbative level, the starred line, is always negative and significantly larger than the partial correction obtained by only including all Higgs diagrams.

Figs. 4 and 5 show the differential distributions in $t \bar{t}$ invariant mass for two Higgs mass values, one just above the on-shell $t \bar{t}$ threshold $\left(M_{H}=370\right.$ $\mathrm{GeV})$ and another well above it $\left(M_{H}=500 \mathrm{GeV}\right)$, respectively. The overall trend here is the same as the one just established at inclusive level, i.e., the salient feature is again that the full $\mathcal{O}\left(\alpha_{S}^{2} \alpha_{W}\right)$ effects swamp those involving the $s$-channel Higgs diagram only. Curiously though, the aforementioned peak-dip structure is maintained (compare the starred histogram with the dotted one), albeit a factor approximately 5(8) larger (and still negative) for $M_{H}=370(500) \mathrm{GeV}$. Therefore, the ultimate effect through the complete $\mathcal{O}\left(\alpha_{S}^{2} \alpha_{W}\right)$ is to swamp the Higgs resonance emerging through $\mathcal{O}\left(\alpha_{S}^{2} \alpha_{W}^{2}\right)$. (Notice that the cross sections in the legends of Figs. 45 are the integrated ones over the entire invariant mass range.)

\subsection{CP-odd Higgs state}

As the benchmark for the CP-odd Higgs boson case we take the MSSM with $\tan \beta=1$ and Supersymmetric matter decoupled. Notice in this case the much reduced width of the Higgs boson, with respect to the SM setup, owing to the absence of $A \rightarrow W^{+} W^{-}$and $Z Z$ decays. Fig. 6] shows the inclusive cross section in its usual components. Here, 


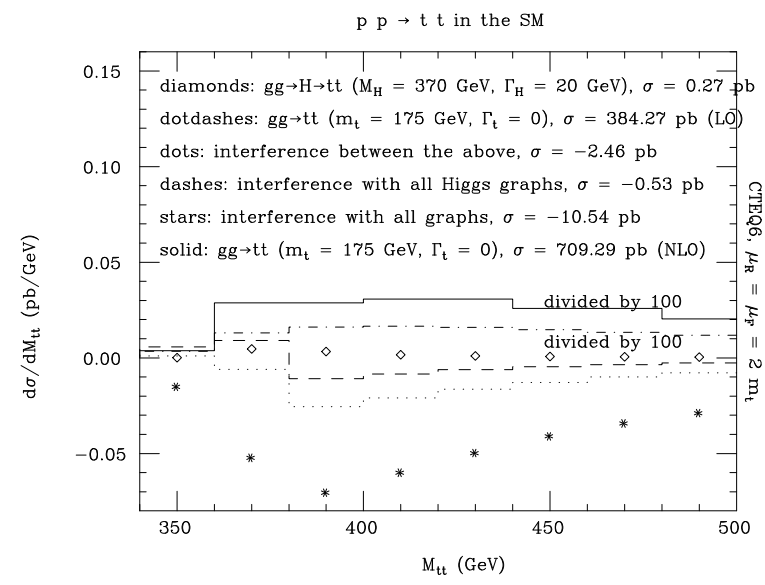

Figure 4: The differential cross section in invariant mass at the LHC for all the components of the $g g \rightarrow t \bar{t}$ process described in the text. The Higgs processes are for the CPeven state of the SM and assume $M_{H}=370 \mathrm{GeV}$. Bin width is $20 \mathrm{GeV}$.

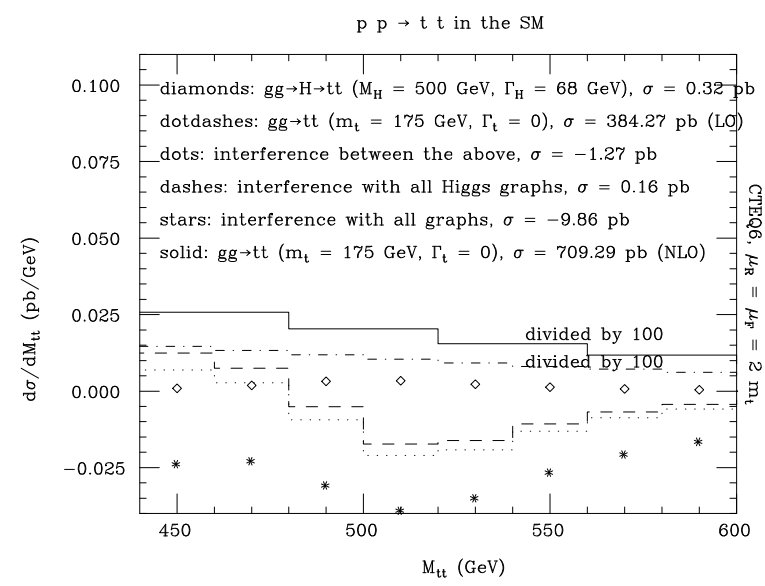

Figure 5: Same as Fig. 4 for $M_{H}=500 \mathrm{GeV}$. since the $\mathrm{BR}(A \rightarrow t \bar{t})$ is much increased compared to the SM case, the $s$-channel Higgs diagram dominates the contributions from other graphs involving a Higgs boson (compare the dotted and dashed curves). Nevertheless, the complete $\mathcal{O}\left(\alpha_{S}^{2} \alpha_{W}\right)$ result is far larger than the terms involving Higgs mediation only, much on the same footing as in the SM.

Figs. 7 and 8 display the usual $M_{t \bar{t}}$ spectra for $M_{A}=370$ and $500 \mathrm{GeV}$, respectively. Again, also for this differential cross section, we see the leading role of the $s$-channel Higgs diagram in the $\mathcal{O}\left(\alpha_{S}^{2} \alpha_{W}\right)$ terms, as it accounts for more than $90 \%$ of the Higgs contributions for $M_{A}=370 \mathrm{GeV}$ (compare dotted and dashed histograms, respectively). However, again in this case, the remainder of the loop diagrams entering the $\mathcal{O}\left(\alpha_{S}^{2} \alpha_{W}\right)$ result is larger. Furthermore, all these terms have always the same (negative) sign, so that they strongly contribute to deplete the yield of the $\mathcal{O}\left(\alpha_{S}^{2} \alpha_{W}^{2}\right)$ signal (compare star and diamond symbols). The situation at $M_{A}=500 \mathrm{GeV}$ is not only qualitatively similar but also quantitatively, as the $s$-channel Higgs contribution is more than $70 \%$ of the total due to Higgs graphs up to $M_{A} \approx 670 \mathrm{GeV}$, after which the interference with the $s$-channel Higgs changes sign. The overall result is that the full $\mathcal{O}\left(\alpha_{S}^{2} \alpha_{W}\right)$ result is again much bigger (a factor of more than 4) than the one due to the $s$-channel Higgs diagram alone. Altogether, again, on sees a strong depletion of the $\mathcal{O}\left(\alpha_{S}^{2} \alpha_{W}^{2}\right)$ Higgs signal once the full $\mathcal{O}\left(\alpha_{S}^{2} \alpha_{W}\right)$ result is accounted for.

\section{Conclusions and outlook}

We have shown that interference effects between LO QCD amplitudes and one-loop diagrams involving weak interactions for the $g g \rightarrow t \bar{t}$ process can be large at the LHC and in fact overwhelming the well studied interference between the former and the component of the latter due to the $s$-channel resonant Higgs diagram only. The overall effect is generally to diminish the significance of the Higgs signal produced as a resonance in the gluon-gluon channel. Therefore, these previously overlooked contributions need to be included in realistic simulations aiming at establishing the LHC potential to extract a Higgs $\rightarrow t \bar{t}$ signature. We have illustrated this at the $14 \mathrm{TeV}$ stage of the LHC using both a CP-even and CP-odd Higgs boson, the former belonging to the SM and the latter to the MSSM. For 


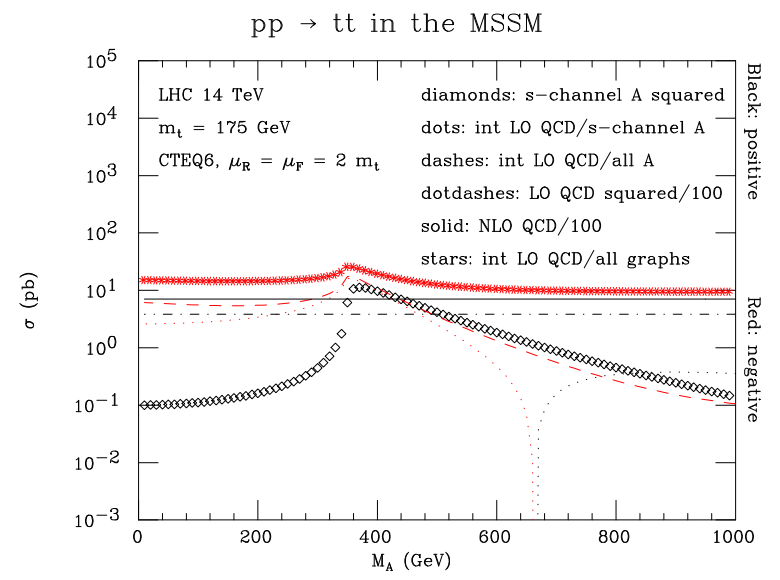

Figure 6: The integrated cross section at the LHC for all the components of the $g g \rightarrow t \bar{t}$ process described in the text. The Higgs processes are for the CP-odd state of the MSSM. Where the curves are red it signifies that they correspond to a negative contribution (notice that $y$-axis is logarithmic).

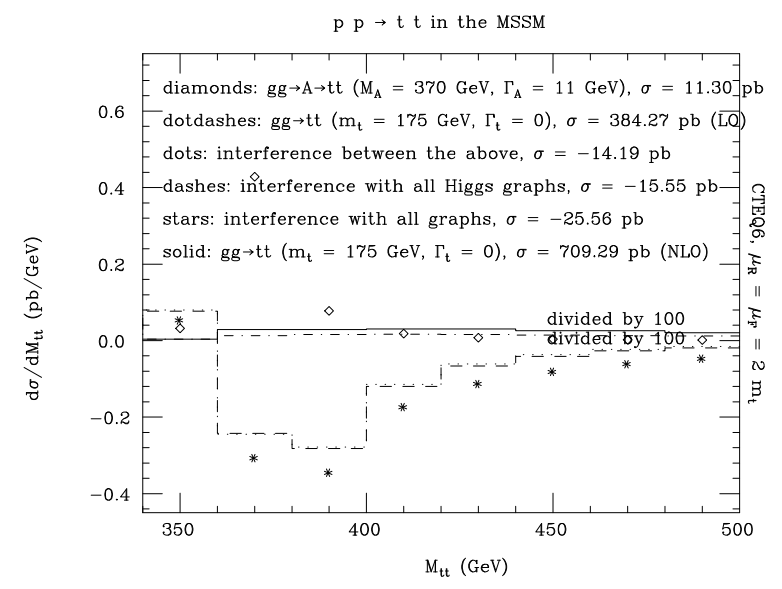

Figure 7: The differential cross section in invariant mass at the LHC for all the components of the $g g \rightarrow t \bar{t}$ process described in the text. The Higgs processes are for the CPodd state of the MSSM and assume $M_{A}=370 \mathrm{GeV}$. Bin width is $20 \mathrm{GeV}$.

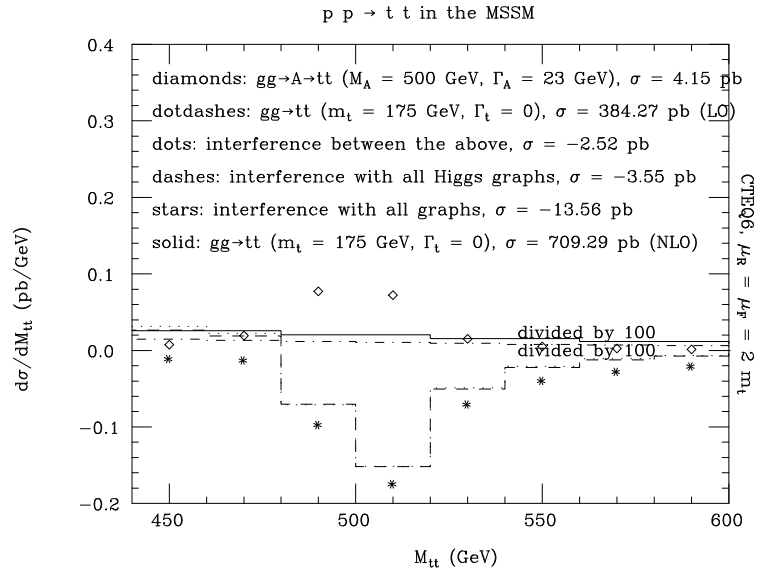

Figure 8: Same as Fig. 7 for $M_{A}=500 \mathrm{GeV}$.

further analyses, we make our program available upon request.

\section{Acknowledgments}

SM is partially supported through the NExT Institute. DAR thanks the Theory Unit at CERN for its hospitality during the preparation of this manuscript.

\section{References}

[1] J.F. Gunion, H.E. Haber, G.L. Kane and S. Dawson, The Higgs Hunter's Guide, Addison-Wesley, Reading MA 1990.

[2] D. Cavalli, D. Froidevaux, F. Gianotti, L. Poggioli, S. Resconi and E. Richter-Was, ATL-PHYS-96-074; ATLGE-PN-74.

[3] ATLAS Collaboration, CERN-LHCC-99-015 (1999) (and references therein).

[4] CMS Collaboration, CERN-LHCC-2006-021 (2006) (and references therein).

[5] A. Djouadi, R. Kinnunen, E. Richter-Was, H.U. Martyn et al., hep-ph/0002258 K. A. Assamagan, M. Narain, A. Nikitenko, M. Spira, D. Zeppenfeld et al., hep-ph/0406152 D. Zeppenfeld, R. Kinnunen, A. Nikitenko and E. Richter-Was, Phys. Rev. D 62 (2000) 013009; U. Aglietti et al., hep-ph/0612172 M. Duhrssen, S. Heinemeyer, H. Logan, D. Rainwater, G. Weiglein and D. Zeppenfeld, Phys. Rev. D 70 (2004) 113009; M. Duhrssen, ATLAS Note PHYS-2003-030; J. Conway et al., hep-ph/0203206

[6] T. Stelzer and S. Willenbrock, Phys. Lett. B 374 (1996) 169; G. Mahlon and S.J. Parke, Phys. Rev. D D53 (1996) 4886 and ibidem 81 (2010) 074024; W. Bernreuther, A. Brandenburg, Z.G. Si and P. Uwer, Phys. Rev. Lett. 87 (2001) 242002 and hep-ph/0410197 P. Uwer, Phys. Lett. B 609 (2005) 271; W. Bernreuther, A. Brandenburg, Z.G. Si and P. Uwer, Nucl. Phys. B 
690 (2004) 81; Acta Phys. Polon. B 34 (2003) 4477; Int. J. Mod. Phys. A 18 (2003) 1357; Phys. Rev. Lett. 87 (2001) 242002 and Phys. Lett. B 509 (2001) 53; A. Brandenburg, Z.G. Si and P. Uwer, Phys. Lett. B 539 (2002) 235; W. Bernreuther, A. Brandenburg and P. Uwer, Phys. Lett. B 368 (1996) 153.

[7] W. Wagner, Rept. Prog. Phys. 68 (2005) 2409.

[8] D. Graudenz, M. Spira and P. M. Zerwas, Phys. Rev. Lett. 70 (1993) 1372

[9] A. Djouadi, P. Gambino and B. A. Kniehl, Nucl. Phys. B 523 (1998) 17; A. Djouadi and P. Gambino, Phys. Rev. Lett. 73 (1994) 2528.

[10] N. Kidonakis and R. Vogt, Phys. Rev. D 78 (2008) 074005; M. Cacciari, S. Frixione, M. L. Mangano, P. Nason and G. Ridolfi, JHEP 0809 (2008) 127; M. Czakon and A. Mitov, Nucl. Phys. B 824 (2010) 111; K. Melnikov and M. Schulze, JHEP 0908 (2009) 049; A. Bredenstein, A. Denner, S. Dittmaier and S. Pozzorini, JHEP 1003 (2010) 021.

[11] P. Nason, S. Dawson and R.K. Ellis, Nucl. Phys. B 303 (1988) 607 and ibidem 327 (1989) 49; W. Beenakker, H. Kuijf, W.L. van Neerven and J. Smith, Phys. Rev. D 40 (1989) 54; W. Beenakker, W.L. van Neerven, R. Meng, G.A. Schuler and J. Smith, Nucl. Phys. B 351 (1991) 507; M.L. Mangano, P. Nason and G. Ridolfi, Nucl. Phys. B 373 (1992) 295.

[12] S. Moretti, M. R. Nolten and D. A. Ross, Phys. Lett. B 639 (2006) 513 [Erratum, ibidem 660 (2008) 607] J. H. Kuhn, A. Scharf and P. Uwer, Eur. Phys. J. C 51 (2007) 37; W. Bernreuther, M. Fucker and Z. G. Si, Phys. Rev. D 78 (2008) 017503 and Int. J. Mod. Phys. A 21 (2006) 914; W. Hollik and M. Kollar, Phys. Rev. D 77 (2008) 014008.
[13] W. Beenakker, A. Denner, W. Hollik, R. Mertig, T. Sack and D. Wackeroth, Nucl. Phys. B 411 (1994) 343; C. Kao, G.A. Ladinsky and C.P. Yuan, Int. J. Mod. Phys. A 12 (1997) 1341; C. Kao and Doreen Wackeroth, Phys. Rev. D 61 (2000) 055009.

[14] K.J.F. Gaemers and F. Hoogeveen, Phys. Lett. B 146 (1984) 347; D. Dicus, A. Stange and S.S.D. Willenbrock, Phys. Lett. B 333 (1994) 126; W. Bernreuther, M. Flesch and P. Haberl, Phys. Rev. D 58 (1998) 114031; R. Frederix and F. Maltoni, JHEP 0901 (2009) 047.

[15] S. Moretti, M.R. Nolten and D.A. Ross, in [12].

[16] J. Pumplin, D. R. Stump, J. Huston, H. L. Lai, P. Nadolsky and W. K. Tung, JHEP 0207 (2002) 012.

[17] J. M. Campbell and R. K. Ellis, Nucl. Phys. Proc. Suppl. 205-206 (2010) 10 (see also http://mcfm.fnal.gov/mcfm.pdf).

[18] S. Moretti and W. J. Stirling, Phys. Lett. B 347 (1995) 291 [Erratum, ibidem 366 (1996) 451].

[19] Z. Kunszt, S. Moretti and W. J. Stirling, Z. Phys. C 74 (1997) 479 arXiv:hep-ph/9611397. 